\title{
Polarization effects in diffraction of light on a planar chiral structure
}

\author{
S. L. Prosvirnin ${ }^{1}$ and N. I. Zheludev 2 , * \\ ${ }^{1}$ Institute of Radio Astronomy, National Academy of Sciences of Ukraine, Kharkov, 61002, Ukraine \\ ${ }^{2}$ School of Physics and Astronomy, University of Southampton, SO17 1BJ, UK
}

(Dated: June 5, 2018)

\begin{abstract}
We analyzed polarization changes of light diffracted on planar chiral array from the standpoint of the Lorentz reciprocity lemma and found the bi-orthogonality in the polarization eigenstates for the waves diffracting though the grating in the opposite direction. Both reciprocal and non-reciprocal component in the polarization azimuth rotation of the diffracted light were identified. The structural chirality of the array arrangement and the chirality of individual elements of the array give rise to polarization effects.
\end{abstract}

PACS numbers: 78.67. -n, 42.25.Jn, 11.30.-j, 71.10Pm, 78.20.Ek

Keywords: planar chirality, nonreciprocity, diffraction

Recently we reported that planar (2D) chiral structures affect the polarization state of light in an enantiomeric fashion, similarly to three-dimensional chiral media 1]. However polarization phenomena on diffraction from planar chiral structures have never been studied theoretically before, leaving the fundamental properties of $2 \mathrm{D}$ chirality not fully understood. Here we report on the results of a theoretical investigation of polarization changes for light diffracted on regular arrays of planar chiral metallic structures from the standpoint of the Lorentz reciprocity theorem. By analyzing propagation of light in two opposite directions we have identified a strong component in polarization effect on diffraction that can be induced either by the chirality of the individual elements of the array, or by arranging non-chiral elements of an array in a chiral fashion.

Let us consider a planar square periodic array of metallic elements of thickness $t$ with equal pitch $d$ along the axes $x$ and $y$ placed between planes $z=0$ and $z=-t$ (see Fig. [1]and Fig. 2). If a plane electromagnetic wave

$$
\mathbf{E}_{i}=\mathbf{A}_{i} e^{-i \mathbf{k}_{i} \mathbf{r}}
$$

of unit amplitude and polarization vector $\mathbf{A}_{i}$ is incident on the array from the region corresponding to $z>0$, the transmitted field may be written as a summation over all diffracted waves, numbered by integer indices $q$ and $p$

$$
\mathbf{E}_{t}=\sum_{q, p=-\infty}^{\infty} \mathbf{a}_{q p} e^{-i \mathbf{k}_{q p}\left(\mathbf{r}+\mathbf{e}_{z} t\right)}, \quad z<-t
$$

where $\mathbf{a}_{q p}$ and $\mathbf{k}_{q p}$ are amplitudes and wave-vectors partial of diffracted waves and

$$
\begin{array}{r}
\mathbf{k}_{q p}=\mathbf{g}+\mathbf{h}_{q p}-\mathbf{e}_{z} \sqrt{k^{2}-\left|\mathbf{g}+\mathbf{h}_{q p}\right|^{2}}, \\
\mathbf{h}_{q p}=2 \pi\left(q \mathbf{e}_{x}+p \mathbf{e}_{y}\right) / d .
\end{array}
$$

Here $\mathbf{e}_{x}, \mathbf{e}_{y}$ and $\mathbf{e}_{z}$ are unit vectors along the axes $x, y$ and $z, \mathbf{g}$ is the component of $\mathbf{k}_{i}$ transverse to the axis $z$ and $k=\left|\mathbf{k}_{i}\right|$. Let us now consider a "reversed" wave with polarization vector $\mathbf{A}_{r}$ approaching the array from the opposite side of the structure $(z<-t)$ along the direction of one of the partial diffracted waves of the "direct" scenario, with indices $s$ and $l$ and the wave vector $\mathbf{k}_{r}=-\mathbf{k}_{s l}$,

$$
\mathbf{E}_{r}=\mathbf{A}_{r} e^{-i \mathbf{k}_{r}\left(\mathbf{r}+\mathbf{e}_{z} t\right)} .
$$

In the region $z>0$ this wave will produce diffracted waves with amplitudes $\mathbf{b}_{q p}$. This corresponds to the "reversed" scenario of diffraction. The Lorentz reciprocity lemma 22 applied to the field superposition in the volume bounded by surface $S$ that consists of planes $x= \pm d / 2$, $y= \pm d / 2, z=z_{1}>0$, and $z=z_{2}<-t$ may be written in the following form:

$$
\oint_{S}\left\{\left[\tilde{\mathbf{E}}_{i} \times \tilde{\mathbf{H}}_{r}\right]-\left[\tilde{\mathbf{E}}_{r} \times \tilde{\mathbf{H}}_{i}\right]\right\} d \boldsymbol{\sigma}=0
$$

where $\tilde{\mathbf{E}}_{i}, \tilde{\mathbf{H}}_{i}$ and $\tilde{\mathbf{E}}_{r}, \tilde{\mathbf{H}}_{r}$ are electric and magnetic fields created by waves incident from opposite directions. By using corresponding field expressions it may be shown from formula (4) that

$$
\sqrt{k^{2}-|\mathbf{g}|^{2}}\left(\mathbf{A}_{i} \cdot \mathbf{b}_{s l}\right)=\sqrt{k^{2}-\left|\mathbf{g}+\mathbf{h}_{s l}\right|^{2}}\left(\mathbf{A}_{r} \cdot \mathbf{a}_{s l}\right) .
$$

The equality (5) constitutes the universal relation between the amplitudes of partial waves in the "direct" and "reversed" diffraction scenarios. Scattering processes are often described in terms of $2 \times 2$ transformation matrices, relating Cartesian components of electric fields in coordinate frames of incident and scattered waves. For the "direct" $(\hat{D})$ and "reversed" $(\hat{R})$ scenarios these matrices for the incident and partial diffracted waves can be introduced as follows: $\mathbf{a}_{s l}=\hat{D} \mathbf{A}_{i}, \mathbf{b}_{s l}=\hat{R} \mathbf{A}_{r}$. It may be shown from equation (5) that these matrices are linearly related and mutually transposed:

$$
R_{n m}=c\left(2 \delta_{m n}-1\right) D_{m n},
$$

where $\delta_{m n}$ is Kroneker index and $c=$ $\sqrt{k^{2}-\left|\mathbf{g}+\mathbf{h}_{s l}\right|^{2}} / \sqrt{k^{2}-|\mathbf{g}|^{2}}$. $\quad$ For the purpose of analyzing the polarization eigenstates of the diffraction 


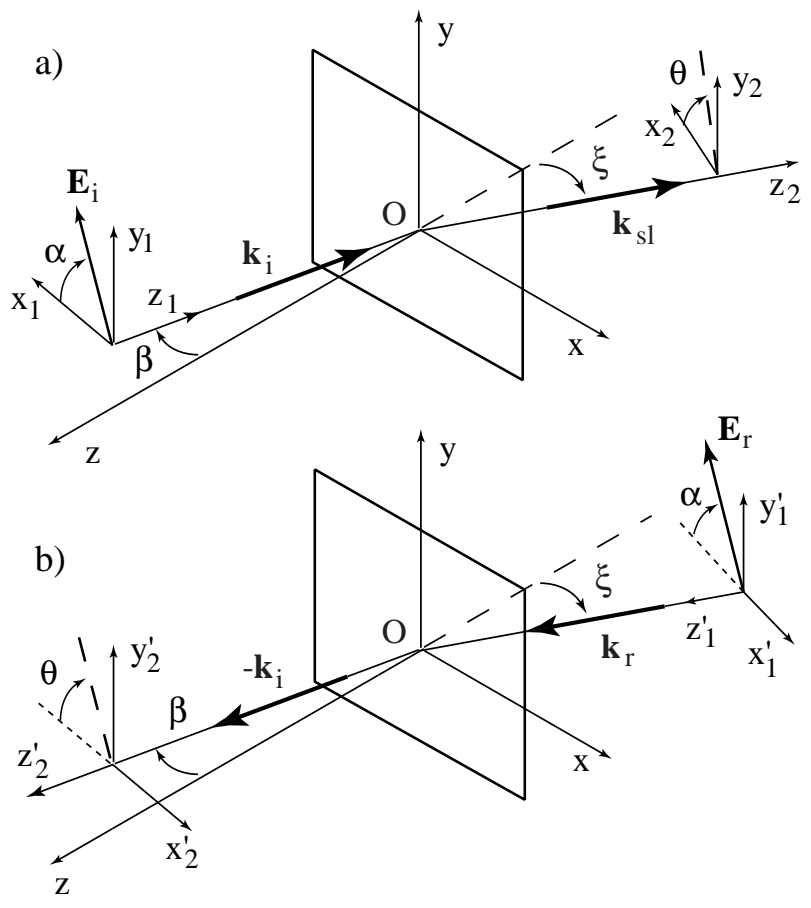

FIG. 1: Coordinate systems and waves in the "direct" (a) and "reversed" (b) diffraction scenarios.

process it is instructive to present both scattering matrices in the coordinate frame of the direct scenario (see Fig. 1,a) where the operator of the reversed scattering process acts on the complex-conjugated field amplitudes. Here the polarization eigenstates are simply two linearly independent eigenvectors of matrices $\hat{R}^{*}$ and $\hat{D}$. The relation between them may be derived from equation (6), which, when converted to the coordinate frame of the direct scenario, gives $R_{n m}^{*}=c D_{m n}^{*}$. It follows from the theory of matrix operators that eigenvectors of the Hermitian-conjugated matrices with elements $D_{n m}$ and $D_{m n}^{*}$, and therefore of matrices $\hat{R}^{*}$ and $\hat{D}$, are biorthogonal or, in terms of polarization eigenstates, are represented by antipode points on the Poincare Sphere, as shown on Fig. 3. In general, the point representing the first eigenstate in the direct scenario $1 d$ is an antipode to one of the points, which represents the eigenstates of the reversed scenario (this point is designed as $2 r$ in Fig. 3). However eigenstate $1 d$ does not necessary coincide with eigensate $1 r$ of the reversed scenario, nor eigenstate $2 d$ coincide with $2 r$. Therefore, the polarization eigenstates in the direct and reversed scenarios presented in the coordinate frame of the direct scenario could be different. Such a situation takes place if the complex matrix $\hat{D}$ is an asymmetric or even a non-diagonal matrix.

We found that scattering matrices of non-zero order diffraction on periodic plano-chiral arrays, where chirality is due to either structural chirality, or chirality of
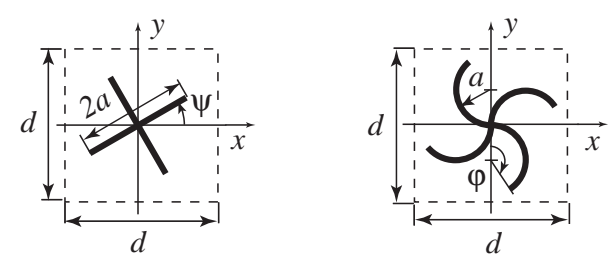

FIG. 2: Structural elements of the arrays: planar straight cross tilted against the array greed on the tilt angle $\psi$, and chiral right-handed gammadion with bending angle $\varphi$.

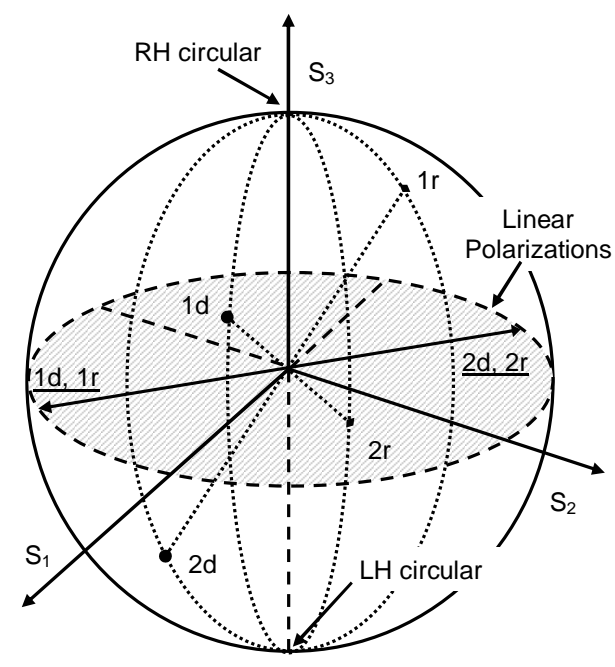

FIG. 3: Schematic representation of diffraction on the Poincaré sphere. For a chiral grating polarization eigenstates in the "direct" scenario $(1 d$ and $2 d)$ and "reversed" scenario $(1 r$ and $2 r)$ are elliptical. Polarization eigenstates (underlined) for non-chiral gratings are mutually perpendicular linear polarization.

individual elements of the array, are either asymmetric or non-diagonal. Below we will illustrate these properties by numerical modelling of the diffraction process for various planar chiral gratings. We calculated the fields and polarization characteristics of light diffracted on gratings numerically using the method described in reference [3]. It is based on a vector integral equation for the surface current induced by the light wave on the array particles. The equation is derived with boundary conditions for ideal metallic structures that assume a zero value for the tangential component of the electric filed on the metal. The integral equation is then reduced to an algebraic equation set by use of the Galerkin technique.

In our modelling we concentrated on planar chiral arrays of 442 symmetry wallpaper group and calculated the polarization eigenstates of the diffraction process and polarization changes occurring in the diffracted wave for different incident polarizations.

We studied diffraction for two different incident angles $\beta$, at $\beta_{1}=0$ and at $\beta_{2}=\arcsin (\pi / k d)$. In the first case, the diffracted wave $(q=1, p=0)$ propagates at an- 
TABLE I: Polarization Eigenstates (PES) for various diffraction processes presented in the "direct" scenario coordinate frame (all angles are measured in degrees, subscripts $d$ and $r$ denote "direct and "reversed" scenarios)

\begin{tabular}{|c|c|c|c|c|c|}
\hline \multirow[b]{2}{*}{ Structure } & \multicolumn{2}{|c|}{ "Direct" Scenario } & \multicolumn{2}{|c|}{ "Reversed" Scenario } & \multirow[b]{2}{*}{ Type of diffraction } \\
\hline & $\begin{array}{l}\text { 1-st PES } \\
\text { (degrees) }\end{array}$ & $\begin{array}{r}\text { 2-nd PES } \\
\text { (degrees) }\end{array}$ & $\begin{array}{l}\text { 1-st PES } \\
\text { (degrees) }\end{array}$ & $\begin{array}{r}\text { 2-nd PES } \\
\text { (degrees) }\end{array}$ & \\
\hline $\begin{array}{l}\text { Straight crosses } \\
\psi=0 ; \beta=0\end{array}$ & $\begin{array}{l}\theta_{1 d}=0.00 \\
\eta_{1 d}=0.00\end{array}$ & $\begin{array}{c}\theta_{2 d}=90.00 \\
\eta_{2 d}=0.00\end{array}$ & $\begin{array}{l}\theta_{1 r}=0.00 \\
\eta_{1 r}=0.00\end{array}$ & $\begin{array}{c}\theta_{2 r}=90.00 \\
\eta_{2 r}=0.00\end{array}$ & $\begin{array}{c}\text { No chiral effect } \\
\theta_{1 d}=\theta_{1 r}, \eta_{1 d}=\eta_{1 r}\end{array}$ \\
\hline $\begin{array}{l}\text { Straight crosses } \\
\psi=+15 ; \beta=0\end{array}$ & $\begin{aligned} \theta_{1 d} & =-14.4 \\
\eta_{1 d} & =-0.05\end{aligned}$ & $\begin{aligned} \theta_{2 d} & =77.1 \\
\eta_{2 d} & =0.08\end{aligned}$ & $\begin{array}{l}\theta_{1 r}=-12.9 \\
\eta_{1 r}=-0.08\end{array}$ & $\begin{array}{l}\theta_{2 r}=75.6 \\
\eta_{2 r}=0.05\end{array}$ & $\begin{array}{c}\text { Chiral effect is present } \\
\theta_{1 d}-\theta_{1 r}=-1.5, \theta_{2 d}-\theta_{2 r}=1.5\end{array}$ \\
\hline $\begin{array}{l}\text { Straight crosses } \\
\psi=-15 ; \beta=0\end{array}$ & $\begin{aligned} \theta_{1 d} & =14.4 \\
\eta_{1 d} & =0.05\end{aligned}$ & $\begin{aligned} \theta_{2 d} & =-77.1 \\
\eta_{2 d} & =-0.08\end{aligned}$ & $\begin{array}{l}\theta_{1 r}=12.9 \\
\eta_{1 r}=0.08\end{array}$ & $\begin{array}{l}\theta_{2 r}=-75.6 \\
\eta_{2 r}=-0.05\end{array}$ & $\begin{array}{c}\text { Chiral effect is present } \\
\theta_{1 d}-\theta_{1 r}=1.5, \theta_{2 d}-\theta_{2 r}=-1.5\end{array}$ \\
\hline $\begin{array}{c}\text { Right gammadions } \\
\varphi=120 ; \beta=0 \\
\end{array}$ & $\begin{array}{c}\theta_{1 d}=6.1 \\
\eta_{1 d}=4.38\end{array}$ & $\begin{aligned} \theta_{2 d} & =-26.6 \\
\eta_{2 d} & =-0.02\end{aligned}$ & $\begin{array}{l}\theta_{1 r}=63.5 \\
\eta_{1 r}=0.12\end{array}$ & $\begin{array}{l}\theta_{2 r}=-83.9 \\
\eta_{2 r}=-4.42\end{array}$ & $\begin{array}{c}\text { Chiral effect is present } \\
\theta_{1 d}-\theta_{1 r}=-57.4, \theta_{2 d}-\theta_{2 r}=57.3\end{array}$ \\
\hline \begin{tabular}{|c|} 
Left gammadions \\
$\varphi=120 ; \beta=0$ \\
\end{tabular} & $\begin{array}{c}\theta_{1 d}=-6.1 \\
\eta_{1 d}=-4.38\end{array}$ & $\begin{aligned} \theta_{2 d} & =26.6 \\
\eta_{2 d} & =0.02\end{aligned}$ & $\begin{array}{l}\theta_{1 r}=-63.5 \\
\eta_{1 r}=-0.12\end{array}$ & $\begin{array}{l}\theta_{2 r}=83.9 \\
\eta_{2 r}=4.42\end{array}$ & $\begin{array}{c}\text { Chiral effect is present } \\
\theta_{1 d}-\theta_{1 r}=57.4, \theta_{2 d}-\theta_{2 r}=-57.3\end{array}$ \\
\hline $\begin{array}{c}\text { Right gammadions } \\
\varphi=120 ; \beta=\beta_{2} \\
\end{array}$ & $\begin{array}{l}\theta_{1 d}=9.9 \\
\eta_{1 d}=5.2\end{array}$ & $\begin{array}{c}\theta_{2 d}=-80.0 \\
\eta_{2 d}=5.2\end{array}$ & $\begin{array}{c}\theta_{1 r}=9.9 \\
\eta_{1 r}=-5.2\end{array}$ & $\begin{array}{c}\theta_{2 r}=-80.0 \\
\eta_{2 r}=-5.2\end{array}$ & $\begin{array}{l}\text { Chiral effect is present } \\
\theta_{1 d}=\theta_{1 r}, \eta_{1 d}=-\eta_{1 r}\end{array}$ \\
\hline \begin{tabular}{|c|} 
Left gammadions \\
$\varphi=120 ; \beta=\beta_{2}$
\end{tabular} & $\begin{array}{l}\theta_{1 d}=-9.9 \\
\eta_{1 d}=-5.2\end{array}$ & $\begin{aligned} \theta_{2 d} & =80.0 \\
\eta_{2 d} & =-5.2\end{aligned}$ & $\begin{array}{c}\theta_{1 r}=-9.9 \\
\eta_{1 r}=5.2\end{array}$ & $\begin{array}{c}\theta_{2 r}=80.0 \\
\eta_{2 r}=5.2\end{array}$ & $\begin{array}{l}\text { Chiral effect is present } \\
\theta_{1 d}=\theta_{1 r}, \eta_{1 d}=-\eta_{1 r}\end{array}$ \\
\hline
\end{tabular}

gle $\xi=\xi_{1}=\arcsin (2 \pi / k d)$ to the array. In the second case the diffracted wave $(q=-1, p=0)$ the same angle makes to the array as the incident wave $\xi=\xi_{2}=-\beta_{2}$ (for definitions of angles see Fig.1). The wave's polarization azimuth $\theta$ and degree of ellipticity $\eta$ were calculated from the Cartesian field amplitudes using the standard definitions: $\tan 2 \theta=s_{2} / s_{1}, \sin 2 \eta=s_{3} / s_{0}$, where $s_{i}$ are the Stokes parameters. The results of our analysis for $d=4 \mu \mathrm{m}, \lambda=2 \pi / k=1520 \mathrm{~nm}, \beta_{1}=0, \xi_{1}=22.3^{\circ}$, $\beta_{2}=11.0^{\circ}, \xi_{2}=-11.0^{\circ}$ are summarized in Table $\llbracket$ and Fig. (4 We considered an array without a substrate. The width of the metal strips was equal to $0.05 \mu \mathrm{m}$.

In optics polarization elements are often classified as reciprocal or non-reciprocal depending on whether their effect on the polarization state of the transmitted light is the same or different for light propagating in the opposite directions. This understanding of optical reciprocity which we will use below is somewhat different from the general, more tolerant definition of reciprocity based on the Lorentz lemma. For the purpose of comparison of the polarization transformations for opposite directions of light propagation, in the table and figures the polarization parameter of the waves are converted into the coordinate frame of the direct scenario. In such a presentation if the values of polarization azimuth rotation in the direct and reversed scenarios are the same, the rotation is truly non-reciprocal. On contrary, a difference between the values of polarization azimuth rotation in the direct and reversed scenarios would represent a reciprocal component of the polarization change, that is analogous to the optical activity effect in a chiral liquid.

The calculations revealed that: i) For all diffraction processes involving twisted or nontwisted arrays, equalities (6) are held to within the numerical accuracy of the method. They are thus compatible with the Lorentz lemma.

ii) For the arrays of straight crosses polarization azimuth rotation in opposite directions have opposite signs due to the difference in the efficiency of diffraction for perpendicular polarization components (line $\mathrm{C}$ on Fig. 4(B)). This non-reciprocity of polarization azimuth rotation is analogous to the polarization rotation nonreciprocity in dichroic media due to anizotropic dissipation.

iii) No polarization rotation is seen in the nondiffracted part of the beam at the normal incidence. It's polarization eigenstates are the same in both directions and for any type of array.

iv) From Table I, one can see that for non-zero order asymmetrical diffraction $(|\xi| \neq|\beta|)$ when individual structural elements of the array are twisted rosettes polarization azimuths of eigenstates for the "direct" and "reversed" scenarios are resolutely different. The difference between the polarization azimuths of the eigensatates depends on the rosette curvature angle $\varphi$ and reaches a maximum of about $57^{\circ}$ at $\varphi=120^{\circ}$. The difference in the eigenstates vanishes at rosette bending angle $\varphi=95^{\circ}$.

v) When individual structural elements of the array are twisted rosettes, polarization azimuth rotation on diffraction has both reciprocal and nonreciprocal components. The non-reciprocal component of the polarization azimuth rotation is due to a difference in the efficiency of diffraction for perpendicular polarization components. 

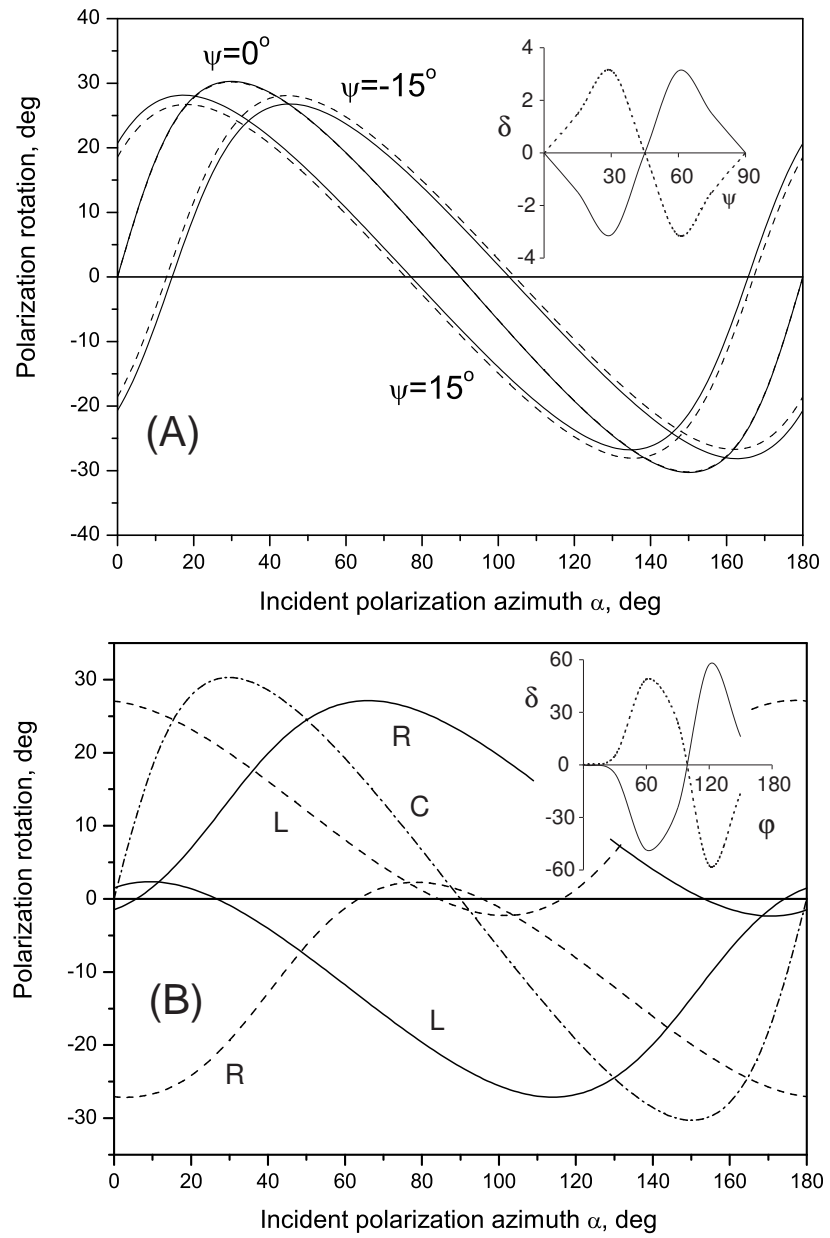

FIG. 4: Polarization azimuth rotation $\Delta=\theta-\alpha$ on diffraction from chiral arrays as a function of incident polarization azimuth, straight line - "direct" scenarios, dashed line - "reversed" scenarios (all results are presented in the direct scenario coordinate frame). The influence of chirality is manifested as a split between corresponding solid and dashed lines: A) Array of straight crosses. The insert shows the chiral difference in polarization azimuth for the two polarization eigenstates as a function of the tilt angle of the crosses; B) Array of left (L) and right ( $\mathrm{R})$ gammadions. The inset shows the chiral difference in polarization azimuth for the two polarization eigenstates as a function of gammadion bending angle.

The corresponding oscillating dependence of the nonreciprocal rotation on the incident angle is shifted in respect of line $\mathrm{C}$ corresponding to straight crosses. It is shifted along the incident polarization azimuth axis to- wards left for left rosettes and towards right for right rosettes. The split between corresponding solid and dashed lines in Fig. 4 indicates the reciprocal component of the polarization azimuth rotation analogous to optical activity.

vi) Nonreciprocity of polarization rotation in the diffraction process is evident when a diffracted light wave is reflected straight back towards the twisted planar structure by a mirror, and then diffracts again. The polarization state of the returning light after the second diffraction is different from that of the incident light, even if the incident light was an eigenstate in the forward direction. For an array of rosettes with $\varphi=120^{\circ}$, the two incident eigenstates and corresponding returning polarizations have azimuths different by $27^{\circ}$ and $21^{\circ}$.

Therefore, polarization effects on diffraction from planar chiral grating can be induced by either structural chirality or the chirality of individual elements of the array. However, no polarization rotation compatible with the Lorentz lemma is possible for a wave transmitting through or reflected from a planar chiral structure at normal incidence, as the scattering matrices are diagonal in this case.

Finally, we shall note that our analysis is underpinned by the Lorentz lemma while our computational method is compatible with it. It shall be noted, however, that the recent observation of broken parity and time reversal evident in polarized optical images of planar chiral structures [4] calls for re-examining the validity of the Lorentz lemma for planar chiral structure.

The authors thank A.Papakostas, A.Potts, D.Bagnall and K.MacDonald for fruitful discussions and acknowledge the support of the Science and Engineering Research Council (UK).

* Electronic address: n.i.zheludev@soton.ac.uk URL: www. nanophotonics.phys.soton.ac.uk

[1] A. Papakostas, A. Potts, D. Bagnall, S. Prosvirnin, H. Coles, and N. Zheludev, Phys. Rev. Lett. 90, 107404 (2003).

[2] J. Kong, Electromagnetic Wave Theory (Wiley, New York, 1986).

[3] S. Prosvirnin, J. of Comm. Techn. and Electr. 44, 635 (1999).

[4] A. Schwanecke, A. Krasavin, D. Bagnall, A. Potts, A. Zayats, and N. Zheludev, ArXiv: cond-mat/0307056 (2 July 2003) (PRL, in press). 